\title{
AUMENTO DA ESTABILIDADE OXIDATIVA DO BIODIESEL DE SOJA ATRAVÉS DE FILTRAÇÃO COM SEMENTE TRITURADA DE MORINGA
}

\author{
D. S. de OLIVEIRA ${ }^{1}$, D. O. MELO ${ }^{2}$, F. R. M. FRANÇA ${ }^{3}$, N. S. LEITE ${ }^{2}$ e G. F. da SILVA ${ }^{1}$ \\ ${ }^{1}$ Universidade Federal de Sergipe, Núcleo de Engenharia de Petróleo \\ ${ }^{2}$ Universidade Federal de Sergipe , Departamento de Engenharia Química \\ ${ }^{3}$ Universidade Federal da Bahia , Departamento de Engenharia Química \\ E-mail para contato: dian.souza@ hotmail.com
}

\begin{abstract}
RESUMO - A produção de biodiesel tem se tornado cada vez mais necessária com a busca por novas fontes de energia que sejam renováveis e limpas. Estudos provam a eficiência e viabilidade do uso do biodiesel como combustível e, com isso, tornando o mesmo alvo de estudos de controle e qualidade. Um dos fatores importantes a ser observado quando se trata da qualidade de um combustível é a sua estabilidade oxidativa, ou seja, sua susceptibilidade à oxidação devido a altas temperaturas ou a exposição com o meio. Partindo de trabalhos que comprovam a alta resistência da moringa a oxidação, o presente estudo avaliou a eficácia do uso de sua semente como um meio filtrante no aumento da estabilidade do biodiesel. Os resultados encontrados se mostraram promissores, com o aumento da resistência do óleo à oxidação sem comprometimento com a qualidade do mesmo.
\end{abstract}

\section{INTRODUÇÃO}

\subsection{O Biodiesel}

Segundo OLIVEIRA (2012), Em vista aos problemas encontrados com o uso de combustíveis fósseis, por serem de fontes não renováveis e do alto grau de poluição que causam, tem-se procurado por formas de energia alternativa e limpa, que não agridam o meio ambiente, que possam ser viáveis e até de menor custo.

Nessa busca, descobriu-se que a mistura de um determinado óleo (de cadeia longa) com um álcool (de cadeia curta) há formação de um éster (esterídeo monoalquil), conhecido também como Biodiesel.

O biodiesel é uma fonte de energia limpa, e renovável, já que para ser produzido é necessário óleo (vegetal) e álcool. A fim de testar a produção de biodiesel através de óleos, como o de milho, soja, girassol ou canola, são feitos testes e experimentos com tais óleos e diferentes álcoois, usando catalisadores em busca de uma maior conversão. 


\section{9 a 22 de outubro de 2014 \\ Florianópolis/SC}

Um dos principais problemas quanto a qualidade do biodiesel produzido é a capacidade que ele tem de oxidar-se quando exposto a determinadas condições (como altas temperaturas). A resistência à oxidação do biodiesel tem ligação estreita com qual tipo de óleo ele foi produzido. Das opções de óleos vegetais encontradas atualmente, uma que tem grande viabilidade é o óleo de soja que, entretanto, apresenta um baixo poder de resistência quanto a oxidação.

Assim, estudos são necessários para que um método de controle de qualidade seja capaz de alterar essa condição do óleo, a fim de melhorar seu potencial anti-oxidante.

\subsection{A Moringa}

Segundo descrições de Pio Côrrea (1984) e Duke (1978), a moringa (Moringa oleifera Lam.) é uma espécie perene, da família Moringaceae e originária do nordeste indiano, sendo amplamente distribuída na Índia, Egito, Filipinas, Ceilão, Tailândia, Malásia, Burma, Pasquitão, Singapura, Jamaica e Nigéria. Ela cresce em regiões de climas variados, desde as subtropicais secas e úmidas, até tropicais secas e florestas úmidas. É tolerante à seca, florescendo e produzindo frutos mesmo em condições de baixas precipitações pluviométricas (Duke, 1978).

Para Dalla Rosa (1993), esta espécie adapta-se a uma ampla faixa de solos, porém se desenvolve melhor em terra preta bem drenada ou em terra preta argilosa, preferencialmente em solo neutro ou levemente ácido. Trata-se de uma planta de múltiplo uso. Quase todas as partes da moringa são ditas como sendo de valor alimentar (folhas, frutos verdes, flores e sementes) e medicinal (todas as partes da planta) (Palada, 1996; Makkar; Becker, 1997).

A rancificação é a decomposição de gorduras, óleos e outros lipídios por hidrólise ou oxidação, ou ambos. Partindo do estudo de PEREIRA (2010), conclui-se que a alta resistência do óleo de moringa à rancificação a torna um composto resistente à oxidação, ou seja, ela apresenta alta estabilidade oxidativa.

Processos de melhoramento na qualidade do biodiesel são importantes e necessários para atender cada vez mais a demanda desse biocombustível no mundo.

Assim, visando aumentar a estabilidade do biodiesel produzido através de diferentes tipos de óleo, procurou-se passar o biocombustível por um meio filtrante constituído da semente da moringa triturada, visto que esta apresenta uma alta estabilidade, a fim de avaliar se tal característica da planta influencia positivamente no aumento da resistência do óleo à oxidação sem que haja alterações na qualidade do mesmo.

\section{METODOLOGIA}

Para realizar o presente estudo, foi usado biodiesel de Soja 100\% puro cedido gentilmente pela usina BiNatural situada em Formosa/Goiás. Foram pré-determinados dois fatores de análise: tempo de mistura antes do processo de filtração e concentração da semente na mistura.

As sementes da Moringa oleífera foram coletadas e devidamente trituradas, como mostra a 
Figura 1.

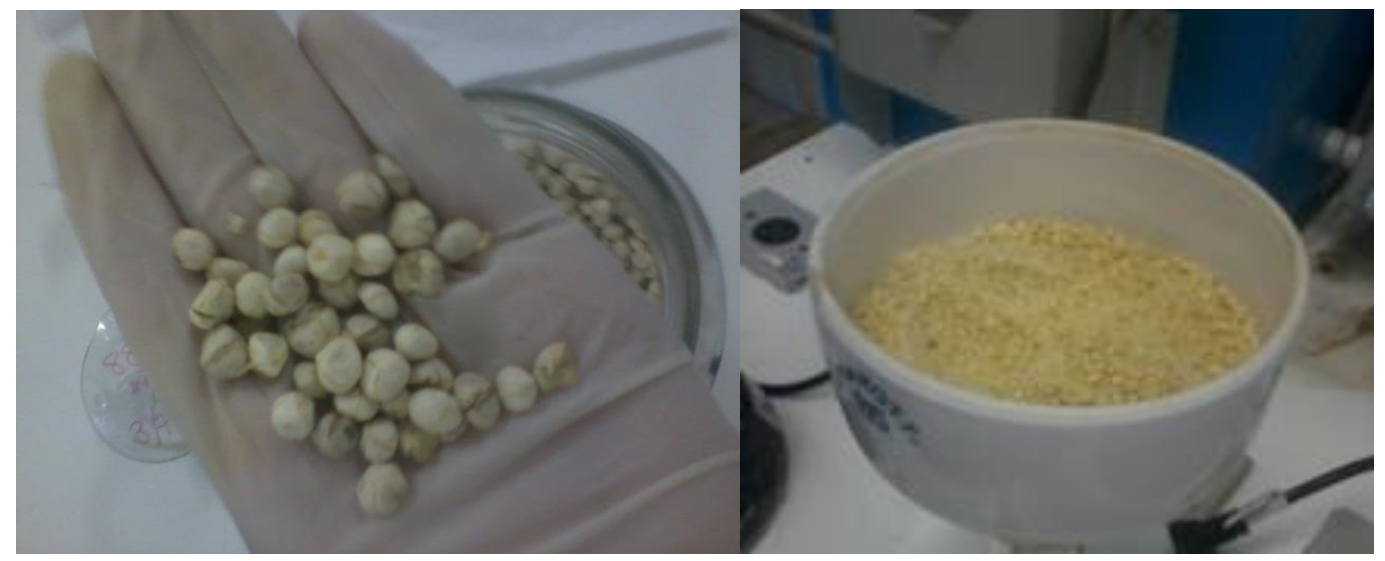

Figura 1: Semente da moringa coletada e posteriormente triturada.

Para cada amostra foram pesados $10 \mathrm{~g}$ do biodiesel e reservados em béqueres isolados da luz e da umidade. Foi definido, como mostra a Tabela 1, um planejamento para separar as amostras em diferentes concentrações de semente e tempo de contato antes da filtração.

$\begin{array}{ccc}\text { Massa de Biodiesel } & \text { Massa de semente } & \text { Tempo de Contato } \\ 10 \mathrm{~g} & 1 \%(1 \mathrm{~g}) & 5 \mathrm{seg} \\ 10 \mathrm{~g} & 1 \%(1 \mathrm{~g}) & 60 \mathrm{seg} \\ 10 \mathrm{~g} & 1 \%(1 \mathrm{~g}) & 300 \mathrm{seg} \\ 10 \mathrm{~g} & 3 \%(3 \mathrm{~g}) & 5 \mathrm{seg} \\ 10 \mathrm{~g} & 3 \%(3 \mathrm{~g}) & 60 \mathrm{seg} \\ 10 \mathrm{~g} & 3 \%(3 \mathrm{~g}) & 300 \mathrm{seg} \\ 10 \mathrm{~g} & 5 \%(5 \mathrm{~g}) & 5 \mathrm{seg} \\ 10 \mathrm{~g} & 5 \%(5 \mathrm{~g}) & 60 \mathrm{seg} \\ 10 \mathrm{~g} & 5 \%(5 \mathrm{~g}) & 300 \mathrm{seg}\end{array}$

Dessa forma, haviam no total 9 amostras a serem analisadas, a qual foram nomeadas da forma arbitrária "Mc,t” (MORINGA concentração, tempo) como mostra a Tabela 2.

Tabela 2. Amostras separadas por concentração e tempo de mistura.

\begin{tabular}{llll} 
& 5seg & 60 seg & 300seg \\
Concentração de 1\%(1g) & $\mathrm{M}_{1,5}$ & $\mathrm{M}_{1,60}$ & $\mathrm{M}_{1,300}$ \\
Concentração de 3\%(3g) & $\mathrm{M}_{3,5}$ & $\mathrm{M}_{3,60}$ & $\mathrm{M}_{3,300}$ \\
Concentração de 5\%(5g) & $\mathrm{M}_{5,5}$ & $\mathrm{M}_{5,60}$ & $\mathrm{M}_{5,300}$ \\
\hline
\end{tabular}


Para o processo de filtragem foi usada uma bomba à vácuo da marca Primar, como mostra Figura 2. Em cada amostra de biodiesel puro era adicionada a devida concentração de semente triturada e agitada no tempo determinado, esse procedimento foi feito apenas imediatamente antes do uso da bomba para filtração à vácuo. A mistura do biodiesel com a semente foi disposta num funil de buchner sobre um papel filtro, e o biodiesel (já sem o material particulado) coletado num Erlemayer.

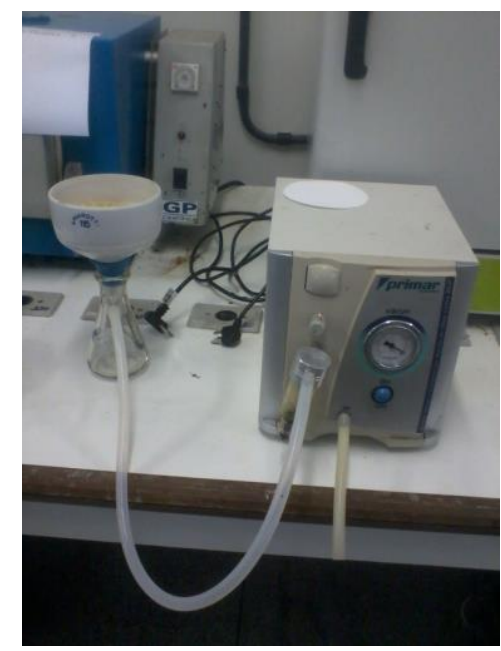

Figura 2. Modelo de filtração á vácuo.

A estabilidade oxidativa (SANTOS, 2008) é definida como a resistência da amostra à oxidação e é expressa pelo período de indução - tempo entre o início da medição e o momento em que ocorre um aumento brusco na formação de produtos da oxidação.

Para analisar a estabilidade dos diferentes tipos de biodiesel (incluindo o biodiesel puro) pesouse $3 \mathrm{~g}$ de cada amostra em tubos de ensaio próprios do 873 Biodiesel Rancimat (Metrohm) (Figura 3) e manteve o ensaio à $110^{\circ} \mathrm{C}$ com fluxo de gás de $10 \mathrm{~L} / \mathrm{h}$.

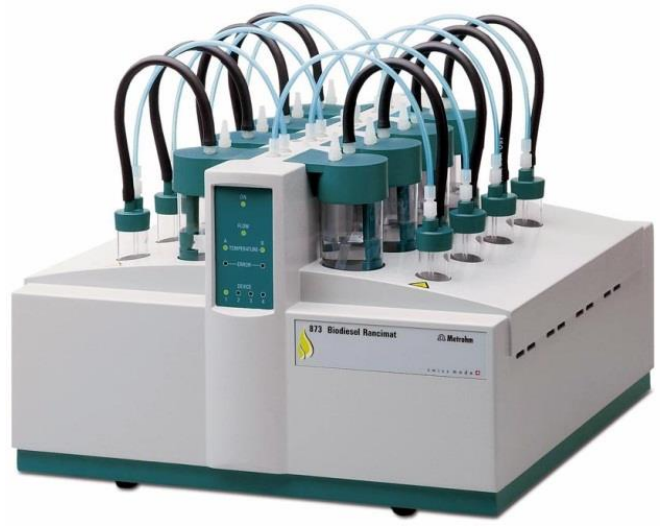

Figura 3. Rancimat para análise de estabilidade.

A fim de avaliar se a qualidade do biodiesel foi alterada durante o processo de mistura e 
consequente filtragem, decidiu-se comparar os dados de índice de acidez (I.A.) do óleo antes e depois da intervenção com a semente.

Índice de acidez (I. A.) é o número de miligramas de $\mathrm{NaOH}$ necessários para neutralizar os ácidos graxos livres de um grama de gordura. Quanto maior o índice de acidez, mais base será consumida. Um elevado índice de acidez indica, portanto, que o óleo ou gordura está sofrendo quebras em sua cadeia, liberando seus constituintes principais, os AG (ácidos graxos livres), e é por esse motivo que o cálculo desse índice é de extrema importância na avaliação do estado de deterioração (rancidez hidrolítica) do óleo ou gordura.

Para a determinação do índice de acidez, em um erlenmeyer de $250 \mathrm{~mL}$, adicionou-se $25,0 \mathrm{~mL}$ de álcool etílico e 1,0 mL de fenolftaleína. Pesou-se 7,05 g do óleo e adicionou-se à solução. Titulouse com hidróxido de sódio 0,1 M até o aparecimento de coloração rósea (a coloração deve persistir por, no mínimo, 30 segundos para que seja considerado o fim da titulação). Anotou-se o volume de base gasto para cada amostra. Calculou-se o índice de acidez (IA) através da Equação 1.

$$
I A=\left(5,61_{x} V_{x} n\right) / p
$$

Onde: $\mathrm{IA}=$ índice de acidez, $\mathrm{V}=$ volume gasto de $\mathrm{NaOH}, \mathrm{n}=$ concentração de $\mathrm{NaOH}$ e $\mathrm{P}=$ peso da amostra (em gramas).

\section{RESULTADOS E DISCUSSÕES}

Os resultados das análises de estabilidade oxidativa obtidos através do Rancimat foram expressos através de gráficos que indicavam o tempo de indução (tempo em que ocorreu a oxidação), os dados estão dispostos na Tabela 3.

Segundo especificações da ANP, para um biodiesel ter qualidade o período de indução na análise de estabilidade deve ser de, no mínimo, 6 horas.

Tabela 3. Tempo de indução para amostras e Biodiesel Puro

$\begin{array}{cc}\text { Amostra } & \text { Tempo de indução (horas) } \\ \text { Bio Puro } & 6,12 \\ \mathrm{M}_{1,5} & 6,08 \\ \mathrm{M}_{1,60} & 6,64 \\ \mathrm{M}_{1,300} & 6,66 \\ \mathrm{M}_{3,5} & 6,69 \\ \mathrm{M}_{3,60} & 7,04\end{array}$




$\begin{array}{cc}\mathrm{M}_{3,300} & 7,3 \\ \mathrm{M}_{5,5} & 7,09 \\ \mathrm{M}_{5,60} & 7,58 \\ \mathrm{M}_{5,300} & 7,94\end{array}$

Com esses dados, construiu-se os gráficos da Figura 4, a seguir, comparando os resultados para cada massa adotada durante o experimento.

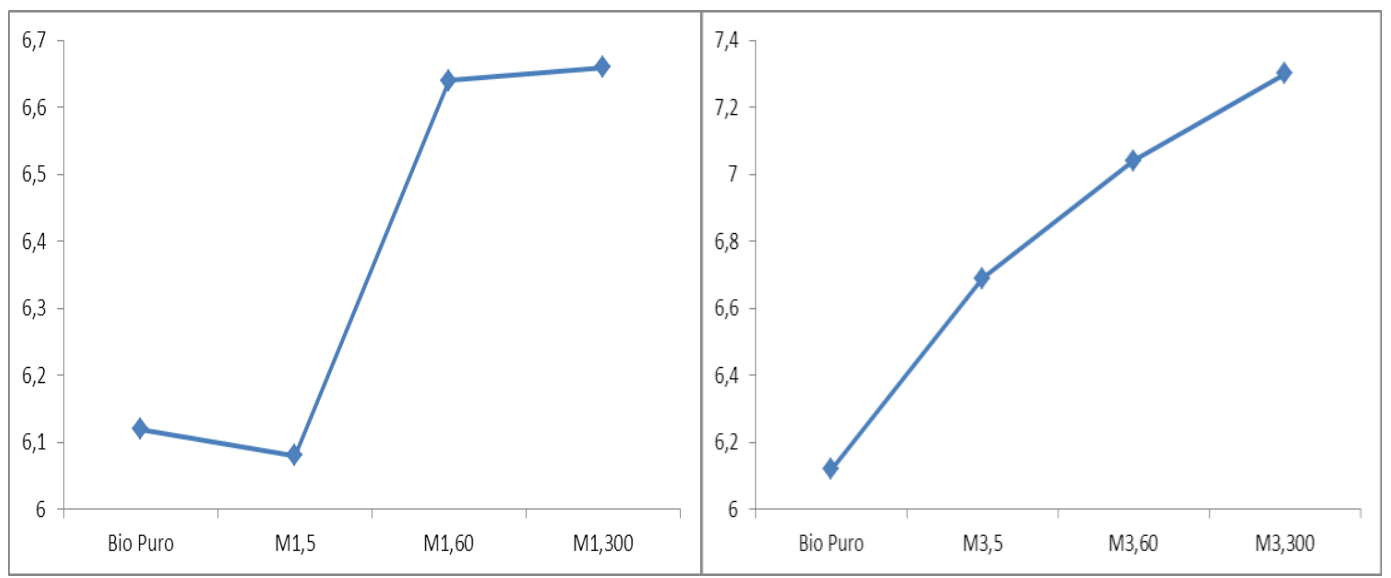

(a)

(b)

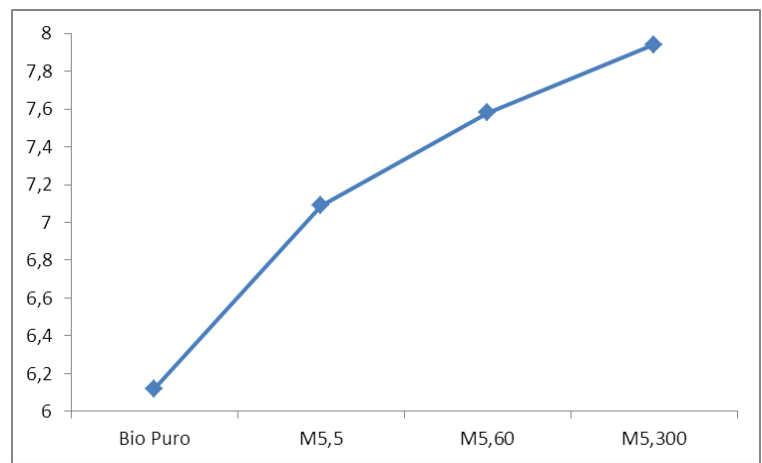

(c)

Figura 4. (a) Crescimento da estabilidade oxidativa para massa de 1g. (b) Aumento da estabilidade para massa de 3g. (c) Resultados para massa e 5g.

Os Resultados do índice de acidez são mostrados na Tabela 4, a seguir. 
Tabela 4 - Índices de acidez das amostras

$\begin{array}{cc}\text { Amostra } & \text { IA } \\ \text { Bio Puro } & 0,0636 \\ \text { M1,5 } & 0,0788 \\ \text { M1,60 } & 0,0795 \\ \text { M1,300 } & 0,0796 \\ \text { M3,5 } & 0,1092 \\ \text { M3,60 } & 0,1098 \\ \text { M3,300 } & 0,1271 \\ \text { M5,5 } & 0,1267 \\ \text { M5,60 } & 0,132 \\ \text { M5,300 } & 0,1113\end{array}$

A norma padrão define que o valor máximo para o índice de acidez (IA) seja de 0,5.

\section{CONCLUSÕES}

Os resultados obtidos demonstram, segundo a Tabela 3, que há um aumento da estabilidade oxidativa do óleo à medida que ele passa pelo processo de filtração com a semente de moringa usada como meio filtrante. Os dados indicam que há um aumento linear, tanto com o aumento do tempo de mistura do óleo com a semente, quanto com o aumento da concentração de semente usada para o processo.

Esse estudo atingiu seu objetivo inicial que era usar um meio natural para aumentar a resistência do combustível à oxidação, o processo se mostra promissor e sem danos à qualidade físico-química do óleo, dados que podem ser comprovados pela Tabela 4. Segundo esta, nota-se que mesmo havendo um aumento da acidez do biodiesel, o índice encontra-se muito abaixo do máximo permitido.

\section{REFERÊNCIAS}

AGÊNCIA NACIONAL DO PETRÓLEO, GÁS NATURAL E BIOCOMBUSTÍVEIS SUPERINTENDÊNCIA DE REFINO E PROCESSAMENTO DE GÁS NATURAL - SRP. Boletim Mensal de Biodiesel, Fevereiro de 2012.

DALLA ROSA, K. R. Moringa oleifera: a perfect tree for home gardens. Hawai: NFTA, Agroforestry Species Highlights, v.1, 2p, 1993.

DUKE, J. A. The quest of tolerant germplasm. In: YOUNG, G. (Ed.) Crop tolerance to subtropical land conditions. Madison. American Society Agronomial Special Symposium, v.32, p.1-16, 1978.

MAKKAR, H.P.S.; BECKER, K. Nutrients and antiquality factors in different morphological parts of 
the Moringa oleifera tree. Journal of Agricultural Science, Cambridge, v.128, p.331-322, 1997.

OLIVEIRA, D. S. Desenvolvimento de um sistema de produção de biodiesel em reator por processo contínuo. Programa Especial de Inclusão em Iniciação Científica - PIIC POSGRAP/PROEST/UFS. 2012.

SANTOS, N. A. dos, Propriedades Termo-oxidativas e de Fluxo do Biodiesel de Babaçu (Orbignya phalerata), João Pessoa, 2008.

PALADA, M.C. Moringa (Moringa oleífera Lam.): a versatile tree crop with horticultural potential in the Subtropical United States. HortScience, v.31, n.5, p.794-797, 1996.

PEREIRA, D. F. Caracterização físico-química do óleo de moringa para possível rota de obtenção de biodiesel, IV Congresso Brasileiro de Mamona e I Simpósio Internacional de Oleaginosas Energéticas, João Pessoa, PB. Pg. 1845. 2010.

PIO CORRÊA, M. Dicionário das plantas úteis do Brasil e das exóticas cultivadas. Rio de Janeiro: MA/IBDF, v.5, p.233-234. 1984. 